\title{
A CFD and Experimental Investigation of the Mixing Process in Compartment Fires
}

\author{
GEORGES GUIGAY ${ }^{1}$, JEAN-MICHEL MOST ${ }^{2}$, JONAS ELIASSON $^{3}$, and BJÖRN KARLSSON ${ }^{3}$ \\ ${ }^{1}$ Mannvit Engineering \\ Grensásvegur 1, 108 Reykjavik, Iceland \\ ${ }^{2}$ Pprime Institute \\ CNRS UPR 3346 /ENSMA/University of Poitiers \\ BP40109 - 86961 Futuroscope, France \\ ${ }^{3}$ Department of Civil and Environmental Engineering \\ University of Iceland \\ 101 Reykjavik, Iceland
}

\begin{abstract}
The mixing of reactants, hot fuel-rich gas and cold air, by a gravity wave, is a complex hydrodynamic process essential in the initiation of hazardous conditions in an under-ventilated compartment fire. The mixing process is first studied by carrying out experiments on a specific enclosure, characterizing the gravity wave by means of particle image velocimetry measurements and fast tomography visualizations. The results are then used to calibrate and validate numerical and CFD models of these experiments. Both numerical and experimental methods permit to determine complex hydrodynamic processes such as Kelvin-Helmholtz instabilities and Van Karman vortices, and to quantify the mixing level in a fire compartment.
\end{abstract}

KEYWORDS: under-ventilated, compartment fires, backdraft, PIV, CFD, mixing, fluid dynamics.

\section{INTRODUCTION}

The work presented in this article is part of a $\mathrm{PhD}$ thesis called "CFD and experimental investigation of under-ventilated compartment fires" [1]. Its objective was to obtain a better understanding of gravity currents $[2-5]$ and the resulting mixing in a fire enclosure. When an under-ventilated fire dies due to lack of oxygen, the enclosed room can remain full of hot unburnt gases. If an opening occurs, for example a window breaks or firefighters open a door to the room, fresh oxygen is carried into it by gravity currents, and mixes with these gases. The mixing of gas with oxygen can create a flammable mixture resulting in ignition and an explosive or rapid combustion called backdraft [6-10]. The deflagration propagates, causing an extreme pressure build-up [11]. The resulting flame travels at a speed of several meters per second. The occurrence and intensity of a backdraft is directly related to the mixing level and the characteristics of the gravity wave. In order to study this crucial problem in fire safety engineering, the following goals were defined:

- To obtain a qualitative and quantitative description of a gravity wave (density driven flow in a gravitational field) entering a suddenly opened hot compartment, for a wide range of temperature differences and two opening configurations.

- To validate experimentally a three-phase flow theory developed by the authors and the average velocities of the flow [2].

- To build a numerical model of these experiments, using the CFD software FDS (Fire Dynamics Simulator) developed by NIST. The model is calibrated and validated against the experimental results.

- To identify, from the experimental and numerical results, the different turbulent structures recognized from papers on turbulence [12-15], that are responsible for the mixing process.

This experimental campaign is a set of 87 experiments. The gravity wave at the door opening, induced by a temperature difference of air inside and outside the enclosure, is characterized using particle image velocimetry (PIV) for velocity field measurements, and fast tomography for flow behavior visualization. The numerical model is created using the CFD software FDS (Fire Dynamics Simulator) developed by NIST. 


\section{DESCRIPTION OF THE EXPERIMENTS}

The experiments were carried out and the apparatus specially designed and built by the author at the Pprime Institute of the CNRS in Poitiers, France. The detailed description of the apparatus, control variables and experimental procedure can be found in Ref. [1].

\section{Experimental Apparatus}

The configuration of the apparatus is given in Fig. 1. The experimental apparatus is a $0.555 \times 0.505 \times$ $0.410 \mathrm{~m}^{3}(\mathrm{~L} \times \mathrm{W} \times \mathrm{H})$ box, insulated using ISOFRAX panels and blankets, and sealed with refractory mastic and glass fiber braid seal. VITROCERAM heat resistant windows are one side of the box and on the floor for flow visualization.

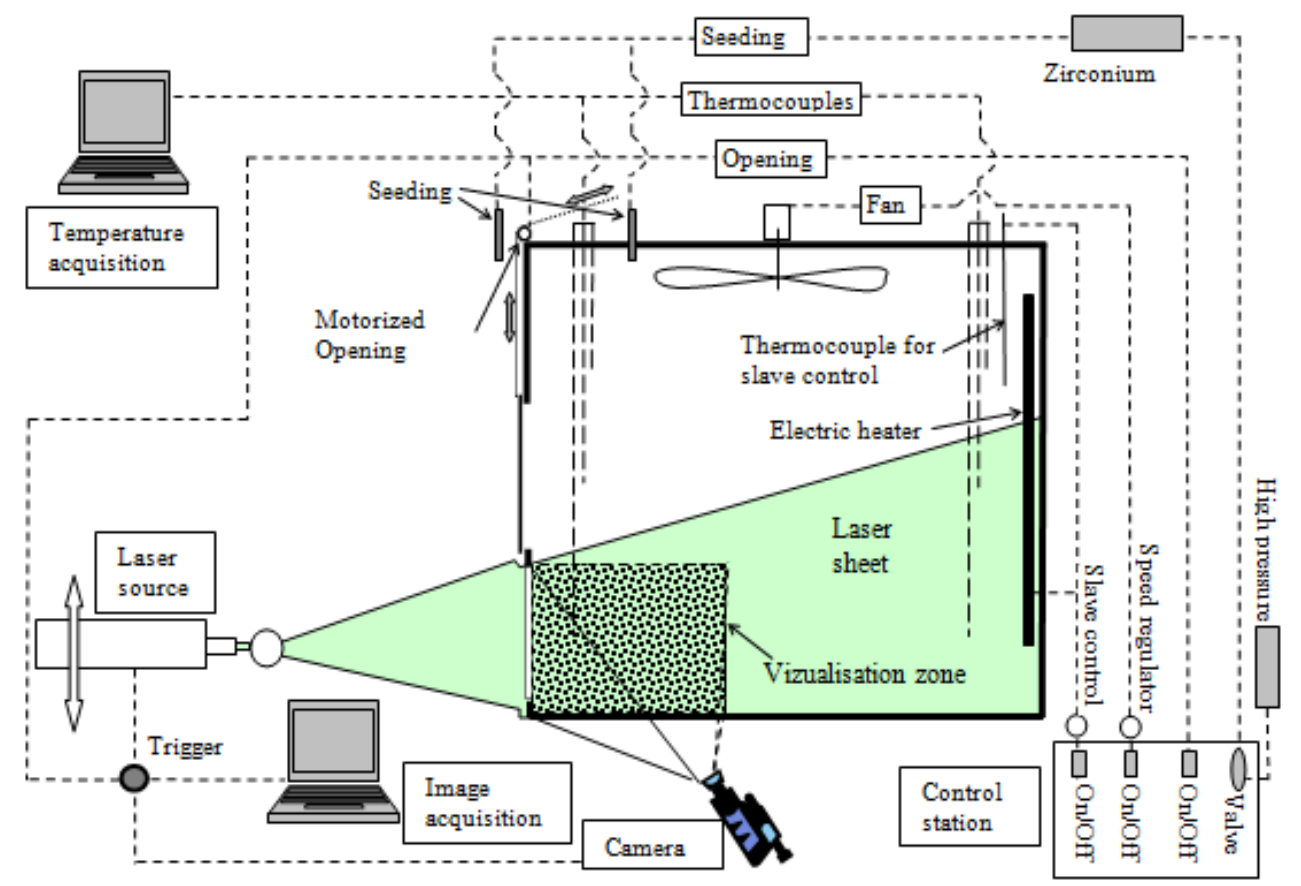

Fig. 1. Description of the experimental apparatus.

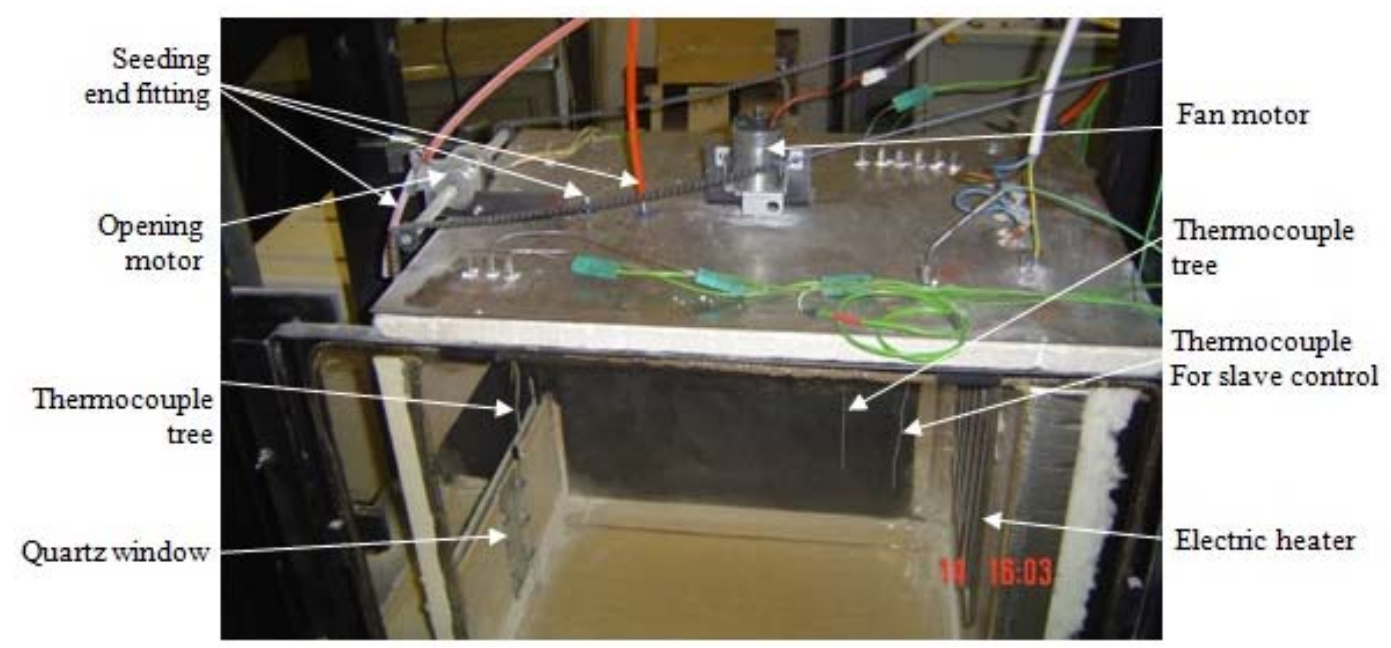

Fig. 2. Picture of the experimental box. 
Before the experiment begins, the box is heated with a $3.2 \mathrm{~kW}$ electric heater slave-controlled with a thermocouple. A fan is installed on the ceiling in order to homogenize the gas temperature. There are two trees composed of three Type-K thermocouples each, one near the window/opening wall corner and one near the diagonally opposite corner, respectively at $0.100,0.250$ and $0.400 \mathrm{~m}$ below the ceiling. The homogeneity of the temperature in both vertical and horizontal direction is checked by thermocouples in the other corners. Thermocouple signals are acquired with a Keithley 2700/2701/2750 Multimeter.

PIV requires the seeding of the flow by micron-sized particles. Due to a temperature in the enclosure over boiling temperature of most liquids, zirconium oxide powder is used. The particle seeder uses an endless screw to push powder in the air flow.

The $0.160 \mathrm{~m}$ opening is covered by a hatch which is lifted using a remotely controlled stepping motor. The opening time is $1 \mathrm{~s}$ and the system does not perturb the incoming flow.

To characterize the behavior of the gravity wave, time-resolved tomography is performed. An argon continuous laser forms a light sheet, and the image is acquired by a fast camera (100 images/s) which is adapted to the time constant of the phenomenon. To determine the wave velocity field, standard PIV measurements are performed. The rate of data acquisition of the PIV pair of images is $2 \mathrm{~Hz}$, which is not completely sufficient to obtain a time resolution of the velocity. The visualizations and PIV recordings are triggered by the hatch opening.

\section{Control Variables and Visualization Panels}

Two types of openings (height $0.160 \mathrm{~m}$ ) are studied, one middle slot and one bottom opening, and eight different inner temperatures $\left(50,75,100,125,150,175,200,225^{\circ} \mathrm{C}\right)$. Figure 3 shows the location of the panels, outside (OU), opening (OP), waterfall behind the middle slot (WF), floor (FL) and rear of the enclosure (BA). The subscripts indicate the type of visualization, i.e. PIV (p) or tomography $(t)$ or both $(p, t)$ for each panel.

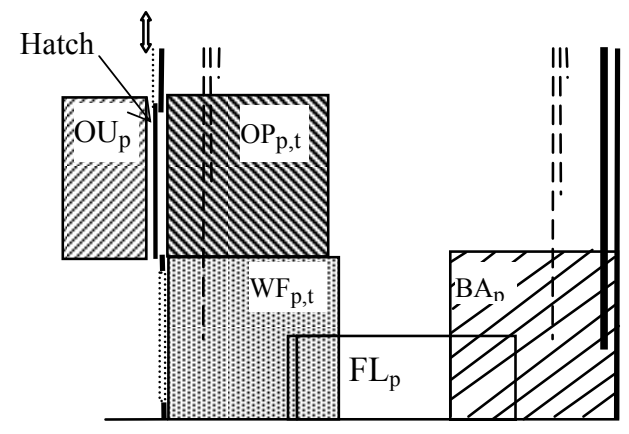

(a)

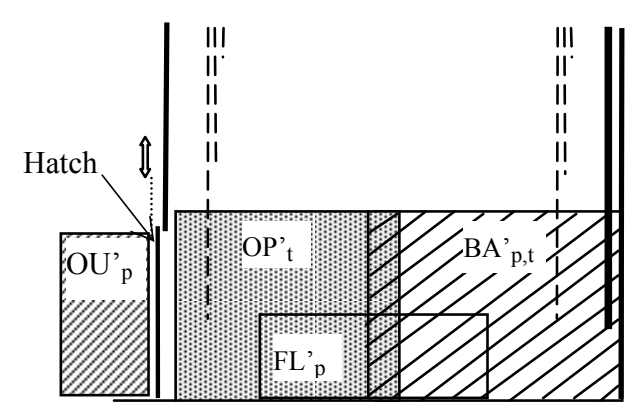

(b)

Fig. 3. Position of the visualization panels: (a) middle; (b) bottom opening configuration.

\section{Experimental Procedure}

To achieve the temperature build-up, the final hot temperature $T_{h}$ is entered into the slave command, and both heater and fan are turned on. The temperature homogeneity is considered acceptable when all of the seven thermocouples are within a range of $T_{h} \pm 10 \%$. The heater and fan are turned off and a $10 \mathrm{~s}$ delay is allowed to get a damping of the convective flow. A puff of air is injected into the seeding system for $2 \mathrm{~s}$ to form a cloud of particles at the enclosure entrance. The hatch is then opened triggering the laser source and the image acquisition. 


\section{EXPERIMENTAL RESULTS}

\section{Quantitative Results - Visualization with Fast Tomography}

Figures 4 to 6 show instantaneous tomography images respectively on panels $\mathrm{OP}_{\mathrm{t}}$ and $\mathrm{WF}_{\mathrm{t}}$ and $\mathrm{OP}_{\mathrm{t}}$ ' for experiments performed with $T_{h}=100{ }^{\circ} \mathrm{C}$. In Fig. 4, only the inside flow is seeded and it shows the formation of a small-scale pair of counter-rotating vortices (Obs 4.1) between cold entering air and hot inside air. The time resolved sequences of images shows successive vortex coalescence process where pairs of Van Karman vortices are ingested into a single large vortex [8]. Other visualizations have shown pulsating disturbances at the opening despite minimizing air disturbance in the room. Figure 5 shows the evolution of the waterfall. A 3-D recirculation zone is formed behind the step (Obs 5.1), and two different free boundary layers creating vortex entrainments can be observed. One is due to the shear friction between the cold layer and the recirculation zone (Obs 5.2) and the other one is due to shear friction between hot and cold layers (Obs. 5.3). The wave detaches itself from the waterfall in a pulsating mode enhancing the flow mixing (Obs. 5.4). Figure 6, for hatch at the floor level, shows the formation of a more standard and steady wave with vortices (Obs 6.1), without any waterfall disturbing the flow, and different stages of cusp entrainment (Obs 6.2). These vortices are typical of Kelvin-Helmholtz instabilities [15] where two fluids in parallel motion with different velocities and densities will yield an unstable interface. This relatively stable flow phase is rapidly affected by the rebounded wave on the rear panel (Obs 6.3). These observations show a thinner mixing zone between the flows. In real cases, the ignition zone must be precisely located to lead to a transition to backdraft. The next step will be to estimate the thickness of the mixing layers by video treatment.

Top of opening

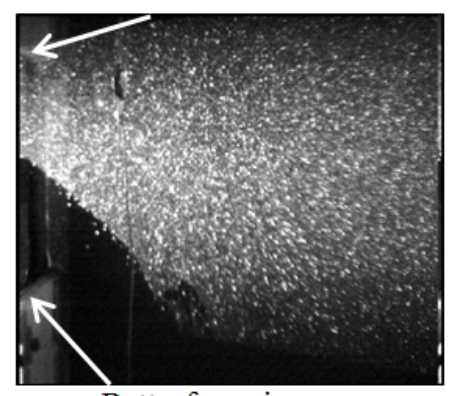

Bott. of opening
Obs 4.1
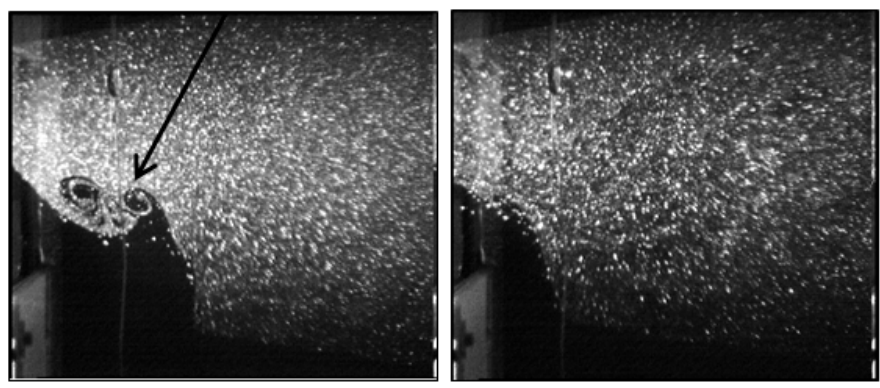

Fig. 4. Tomography on panel $\mathrm{OP}_{\mathrm{t}}\left(T_{h}=100^{\circ} \mathrm{C}\right)$, after $2,4.2$ and $6.4 \mathrm{~s}$ (left to right).

Bott. of opening Obs 5.3

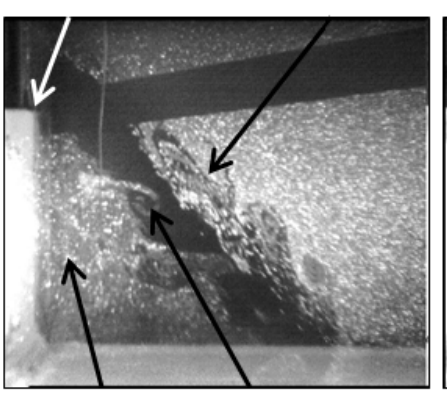

Obs 5.1
Obs 5.2

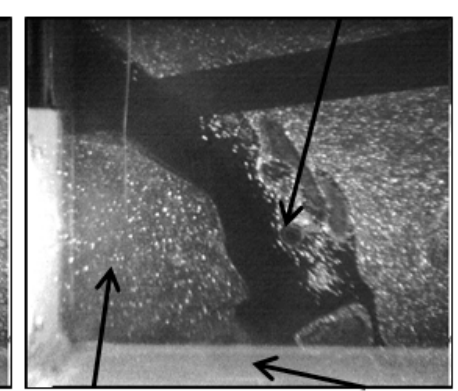

Obs 5.1

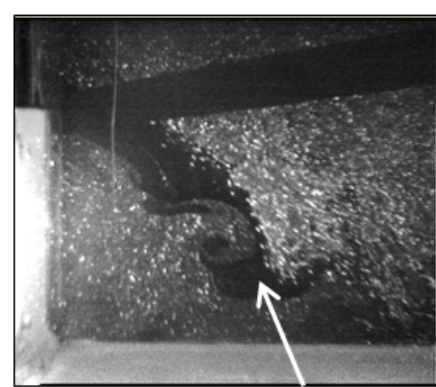

Obs 5.4

Fig. 5. Tomography on panel $\mathrm{WF}_{\mathrm{t}}\left(T_{h}=100^{\circ} \mathrm{C}\right)$, after $2.2,4.6$ and $6.4 \mathrm{~s}$ (left to right). 

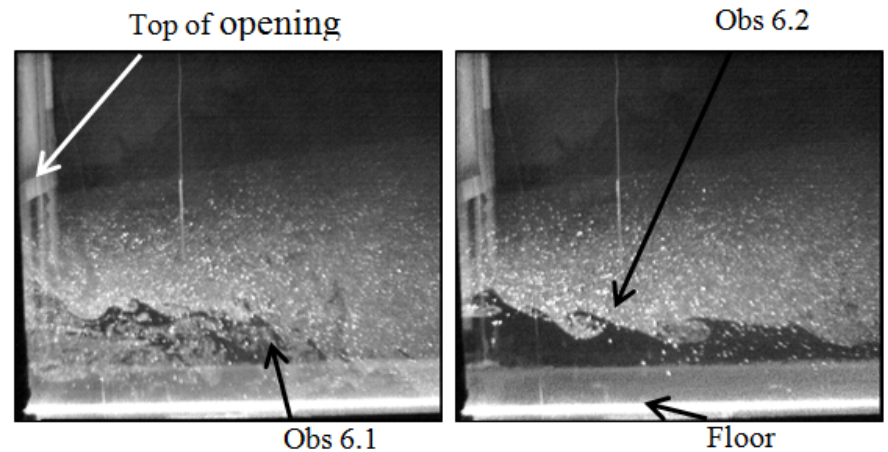

Floor

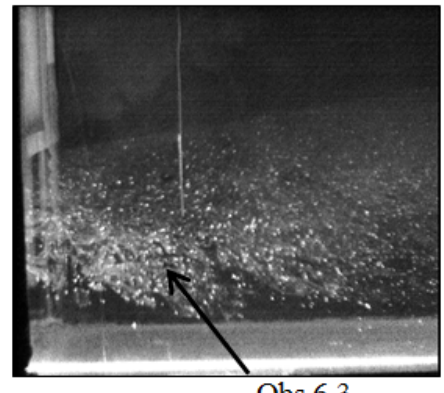

Fig. 6. Tomography on panel $\mathrm{OP}_{\mathrm{t}}{ }^{\prime}\left(T_{h}=100^{\circ} \mathrm{C}\right)$, after $1.2,2.4$ and $4.2 \mathrm{~s}$ (left to right).

\section{Quantitative Results - Velocity Measurements by Particle Image Velocimetry}

Figure 7 shows an instantaneous velocity field at the opening, obtained by Particle Image Velocimetry.

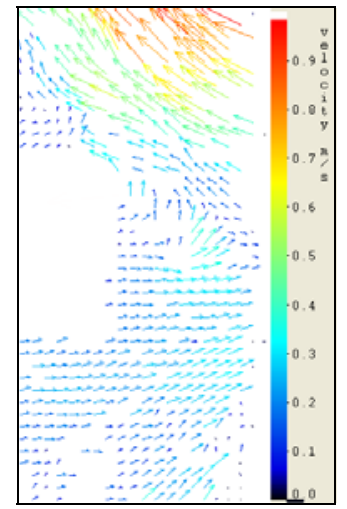

Fig. 7. Instantaneous velocity field at $\mathrm{OU}$ (opening) after $6.5 \mathrm{~s}\left(T_{h}=200{ }^{\circ} \mathrm{C}\right)$.

The mean velocity at the opening can be determined with a statistical calculation. The thickness of the mixing zone of the input flow at the interface is determined allowing characterization of the input air mass flow rates for various $T_{h}$ values. This information is essential to validate analytical and numerical models developed in Refs. [2] and [3]. The input air mass flow rate controls the flow Reynolds number and the shear stress between the flows, hence the cold and hot air mixing. The analytical models are based on classic hydraulic equations, taking into account the shape of the opening and the mixing at the interface by determination and introduction of correction factors $C$ and $\alpha$. This gives, at stable flow, the expression for the average velocity of the hot layer derived by the authors in Ref. [3]:

$$
V_{h}=C_{h} \sqrt{\frac{1}{\alpha_{h}} \Delta_{h} g\left(1-\frac{1}{1+\left(\rho_{h} / \rho_{c}\right)^{1 / 3}}\right)} h
$$

where $\rho$ is the density, $\Delta_{h}$ the dimensionless reduced mass $\left(\Delta_{h}=\left(\rho_{c}-\rho_{h}\right) / \rho_{h}\right)$ and $\mathrm{h}$ is the height of the opening. The subscripts stand for hot or cold layer.

Figure 8 shows the evolution and mean longitudinal component of the velocity for $T_{h}=75^{\circ} \mathrm{C}$ and $200{ }^{\circ} \mathrm{C}$. It shows an accelerating phase just after full opening (from 3 to $7 \mathrm{~s}$ for $T_{h}=75{ }^{\circ} \mathrm{C}$ and 3 to $5 \mathrm{~s}$ for $T_{h}=200^{\circ} \mathrm{C}$ ). The velocity fluctuations are damped after a few seconds and reach a constant value function of the $T_{h}$ values which allows the comparison between analytical and experimental average velocities. This last result shows a good agreement between experimental and analytical results, and tends to confirm the three phases flow theory developed in Ref. [2]. 


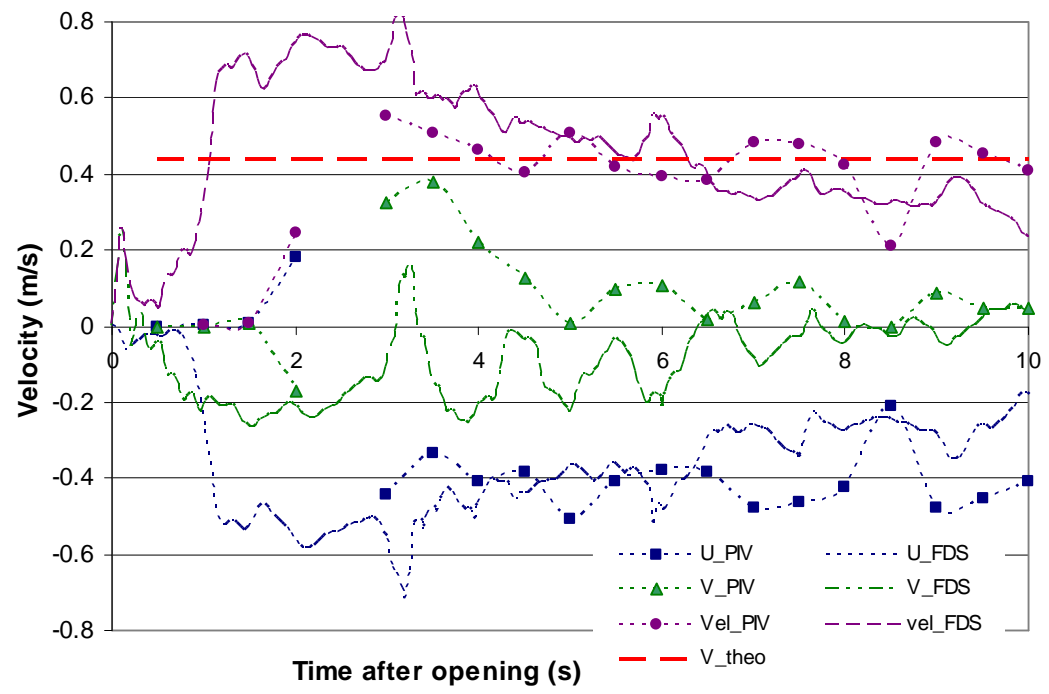

Fig. 8. Velocity history at the opening for $T_{h}=200{ }^{\circ} \mathrm{C}$ from PIV measurements, FDS simulation and average velocity from analytical study.

\section{DESCRIPTION OF THE FDS NUMERICAL MODEL}

A numerical model of the experiments was set up using Fire Dynamics Simulator (FDS 5, [16,17]). The geometry of the model is shown in Fig. 9. The dimensions of the domain are $0.8 \times 0.205 \times 0.7 \mathrm{~m}^{3}(\mathrm{~L} \times \mathrm{W} \times$ $\mathrm{H}$ ) with a mesh composed of $160 \times 41 \times 140=918400$ cells regularly spaced (spatial resolution $=5 \mathrm{~mm}$ ). The domain is extended in front and on top of the compartment, in order to simulate the flow correctly and prevent the pressure boundary conditions from influencing the flow inside the compartment. Only half of the compartment is modeled by using the symmetry of the compartment, applying the "MIRROR" function to the plan $y=0.205 \mathrm{~m}$, the same plan as the laser sheet in the experiments. A sensitivity analysis has been carried out to test the dependence on the grid size and the validity of using the mirror function in the problem. As everything is symmetrical (geometry, boundary and initial conditions), the tests showed no significant differences, and using a $5 \mathrm{~mm}$ mesh resolution and the mirror function led to a stable solution. In order to simulate the opening, the hatch is composed of 32 removable obstacles. One obstacle is removed every $0.032 \mathrm{~s}$, from the bottom to the top of the opening. The total opening time is $1.024 \mathrm{~s}$, which is very similar to the experiments.

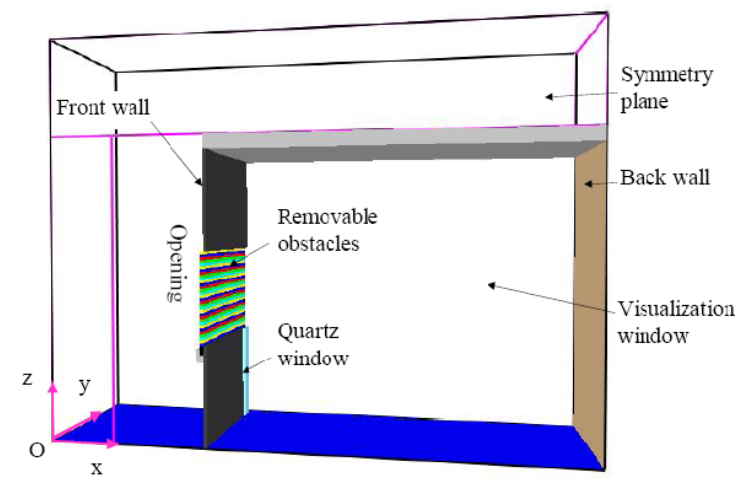

Fig. 9. FDS model of the experimental apparatus (middle opening configuration).

The initial conditions specified are the required hot temperature inside the compartment and ambient $\left(20^{\circ} \mathrm{C}\right)$ outside. The same control variables (i.e. opening configuration and inner temperatures) and the same referencing system as in the experiments is used, e.g. FDS-B-T100 stands for the FDS run in bottom configuration and $T_{h}=100{ }^{\circ} \mathrm{C}$. A complete description of the model is given [1], where its advantages and disadvantages are also discussed. 


\section{COMPARISON OF CFD, ANALYTICAL AND EXPERIMENTAL RESULTS}

\section{Qualitative Results}

Qualitative results discussed earlier concerning tomography permitted the identification of very characteristic turbulent structures. The simulation shows the same vortex coalescence process where a pair of counter-rotating vortices are ingested by a single large vortex discussed earlier (see Fig. 10). These vortices are roughly the same size, although, in the simulation, they first appear further inside the compartment, as indicated by the distances from the bottom of the opening to the center of the structure. In both cases, these vortexes are then ingested into a single larger vortex.

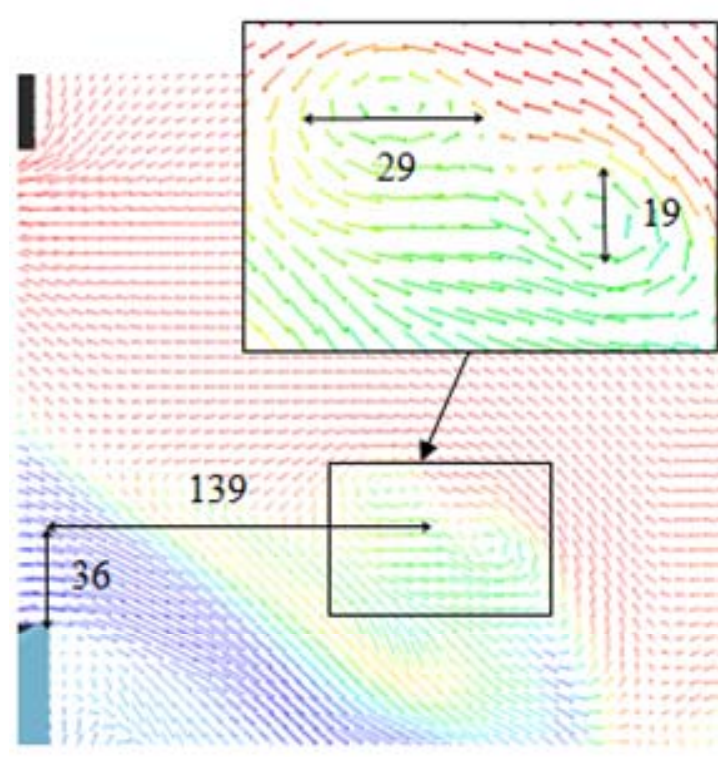

(a)

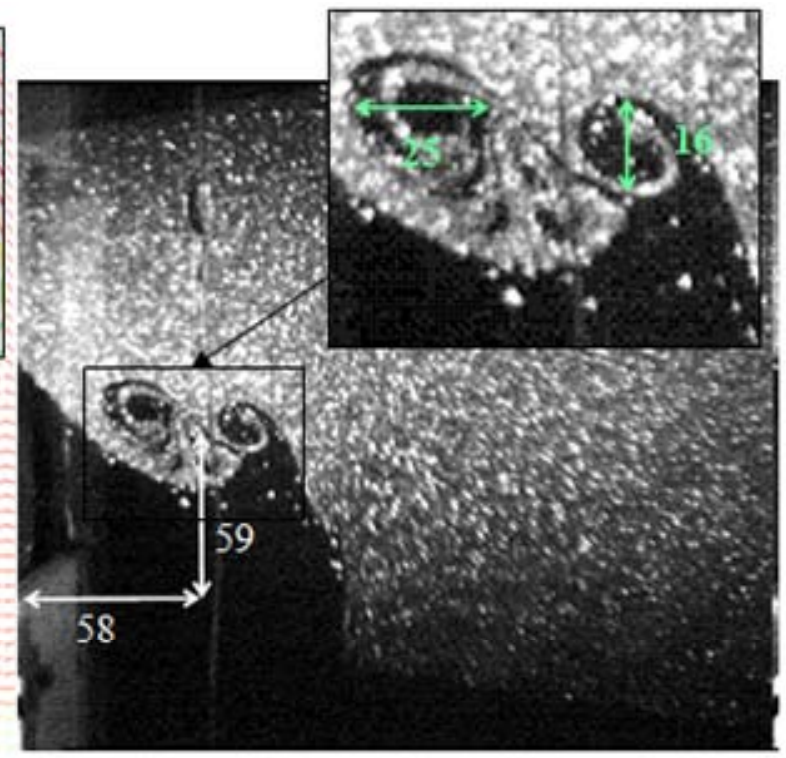

(b)

Fig. 10. View of the flow on panel OP for $T_{h}=100{ }^{\circ} \mathrm{C}$ for: (a) simulations after $3.5 \mathrm{~s}$; (b) tomography after $2.05 \mathrm{~s}$. The position of the pair of vortices is shown relative to the bottom of opening, and their sizes are indicated on the zoomed views.

An interesting structure shown by both simulations and experiments is the "mushroom head" shape of the head of the current before it hits the bottom of the box, on the waterfall visualization panel WF (see Fig. 11). On each side of the head is a rotating structure creating this particular shape, characteristic of a stationary jet flow. The vortex on the internal side of the waterfall is roughly the same size in the simulation and experiments. On the external side of the waterfall, the simulation shows cusp entrainment with a well-mixed structure which is a bit different from the vortex entrainment shown with the experiments. In this case, as the cold fluid is going up, its kinetic energy is transformed in potential energy. The cold fluid therefore has less kinetic energy for mixing, which explains the very clear boundary between hot and cold fluid within the vortex. The vortex entrainment creates a significantly smaller structure that the cusp entrainment, which is due to the difference in the mixing efficiency. However, both types of entrainment create significant momentum exchange and mixing. The flow of the wave immediately after the head and up to the opening is significantly wider in the experiments. There is a strong entrainment of hot fluid into these originally cold vortices. The behavior and the influence of these turbulent structures will be discussed later in this article. 


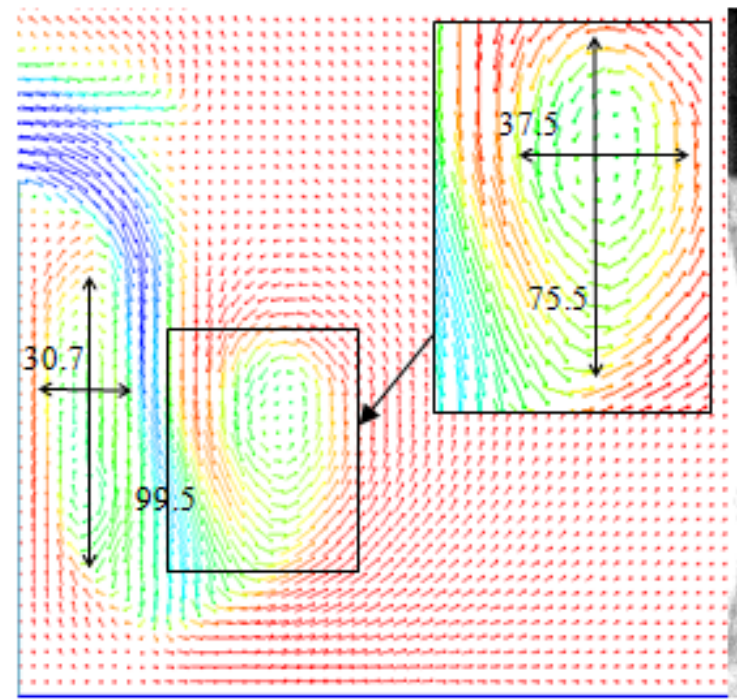

(a)

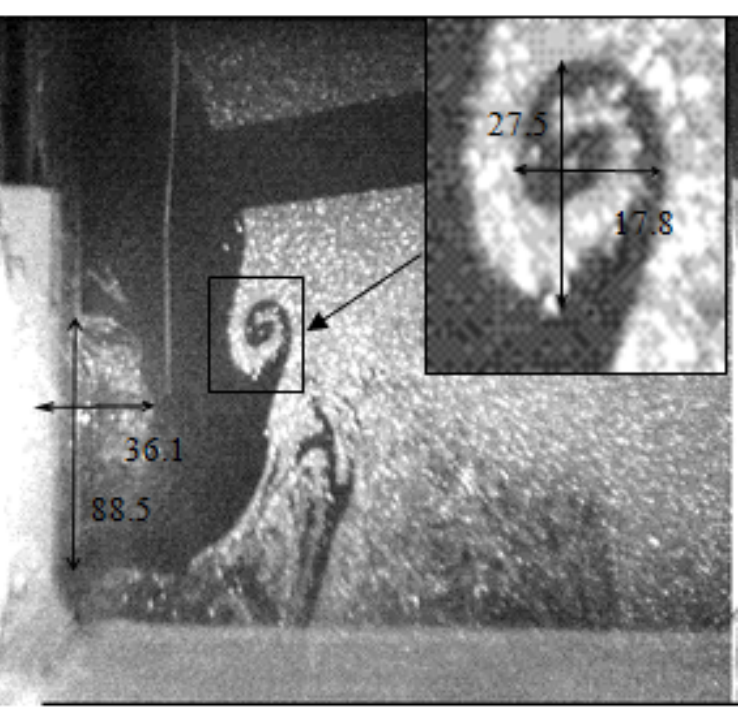

(b)

Fig. 11. View of the flow on panel WF for $T_{h}=50{ }^{\circ} \mathrm{C}$ : (a) after $1.45 \mathrm{~s}$ for simulations; (b) $2 \mathrm{~s}$ for tomography. The position of the pair of vortices is shown relative to the bottom of opening, and their sizes are indicated on the zoomed views.

The experiments and simulations show similar characteristic turbulent structures, as shown in Figs. 10 and 11. The evolution in time shown in Ref. [1] also shows a similar behavior between the experiments and simulations. From a qualitative point of view, it can be concluded that there is a fair comparison between experiments and simulation.

\section{Quantitative Results}

In order to validate the results of the numerical model in a quantitative way, significant features of the gravity wave such as transit times, interface height, velocity at the opening and inside the compartment, are compared with the experimental results, obtained by means of tomography and PIV

The transit time is defined as the time for the leading edge of the gravity current to reach the wall opposite the opening (see Fig. 12). This is evaluated by observing pictures from PIV and FDS in the middle opening configuration and, from PIV, FDS and tomography in the bottom opening configuration. The different results generally show good agreement. In both the middle and the bottom opening configuration, the transit time is shorter in the simulations. This difference might be due to the higher mixing level in the experiments, consequently slowing the gravity current. It might also be due to the fact that the temperatures are not perfectly homogeneous in the experiments, with colder gases at the bottom, reducing the density difference. 


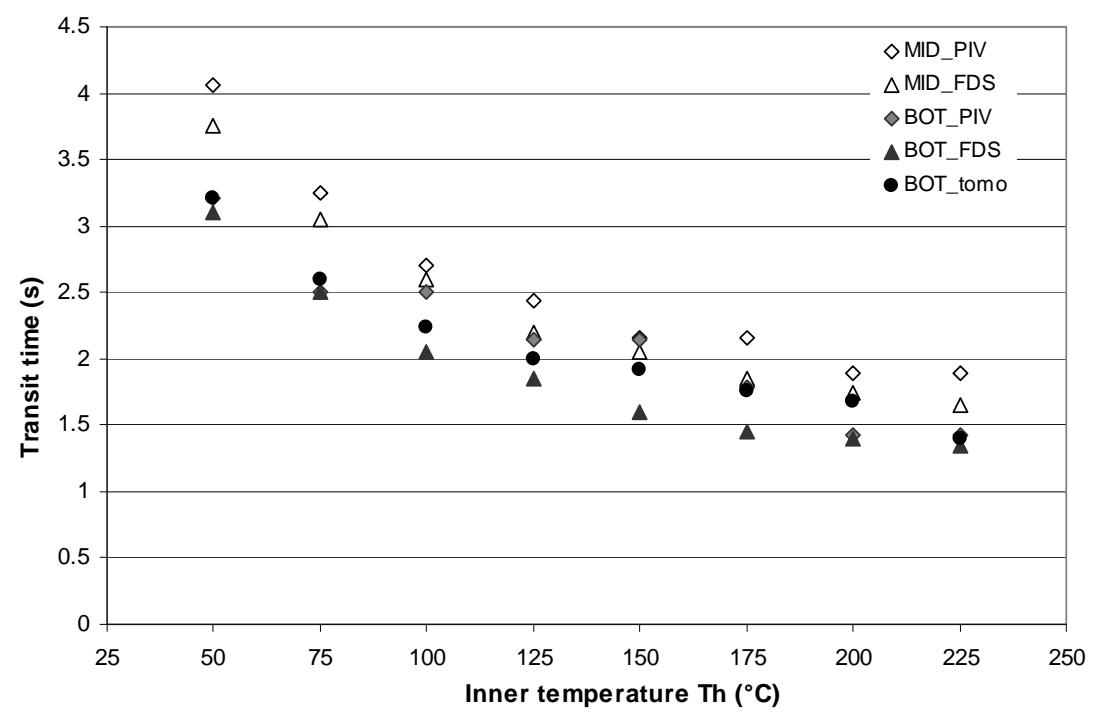

Fig. 12. Transit time for middle (PIV and FDS simulation) and bottom opening configuration (PIV, FDS simulation and tomography).

Figure 8 shows the evolution and mean longitudinal component of the velocity ( $\mathrm{U}$ and $\mathrm{W}$ stand for the horizontal and vertical velocity respectively and Vel stands for its norm) of the hot layer at the opening for $T_{h}=200{ }^{\circ} \mathrm{C}$. It shows an accelerating phase just after full opening (from 3 to $7 \mathrm{~s}$ for $T_{h}=75{ }^{\circ} \mathrm{C}$ and 3 to $5 \mathrm{~s}$ for $T_{h}=200{ }^{\circ} \mathrm{C}$ ). The velocity fluctuations are damped after a few seconds and reach a constant value function of the $T_{h}$ values which allows the comparison of analytical and experimental average velocities. There is a fair agreement between the results for the three methods, analytical, numerical and experimental.

The velocity profiles for the hot layer at the opening were studied in detail at different time steps [1]. Fleischmann et al. [4] first observed a particular shape of the velocity profile. Guigay et al. suggested a profile similar to potential flow in a 180 degree bend around a wall end [3], as shown in Fig 13.

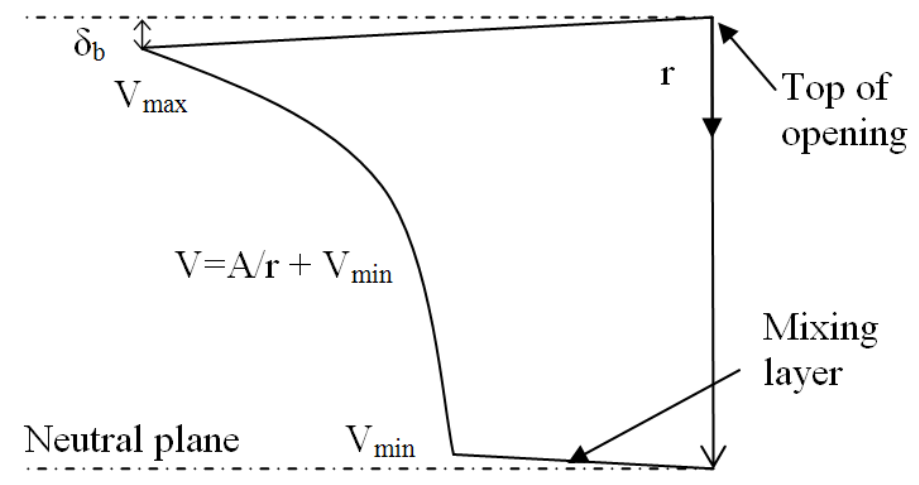

Fig. 13. Details of the shape of the outflow at the opening, showing a potential flow profile.

\section{Discussion on the Model Validation}

Qualitative observations of results from tomography and from the numerical model show that the flow is very unsteady, with turbulent structures creating an offset in both time and position of the interface. These structures are very similar, even though tomography shows vortex entrainment when the simulations also show cusp entrainment (see Figs. 4 and 5). Both create a momentum exchange, but cusp entrainment is more efficient in the mixing process.

Quantitatively, the unsteady behavior, shown by the variation of the interface position, velocity histories both at the opening and inside the compartment makes it difficult to consider velocities averaged over a certain range of time after opening. However, these velocity histories seem to confirm a three phase 
behavior of the flow, with an accelerating phase, followed by a quasi-steady phase and finally a decaying phase. It is possible to extract a significant mean longitudinal velocity for experimental, numerical and analytical results. The agreement between the three methods is considered to be fair for this mean longitudinal velocity at the opening.

There is generally a fair agreement between analytical, experimental and numerical results, validating the transport equations of the FDS numerical model in such situations.

\section{ANALYSIS AND QUANTIFICATION OF THE MIXING PROCESS}

\section{Middle Opening Configuration}

In this part, we follow the evolution of the flow just after the opening. In the case shown in the following figures, the initial hot temperature is $T_{h}=125^{\circ} \mathrm{C}$, but note that the same structures and the same behavior law are observed for every run within the studied temperature range.

Just after the opening, at $t=0.7 \mathrm{~s}$, the head of the flow entering the compartment is a mushroom-shaped structure typical of a stationary jet (see Fig. 14 (a)). This structure, sometimes also referred to as "sinking bubbles" in fluid mechanics, is composed of two counter-rotating coherent structures, one on each side of the flow. Figure 11 shows the velocity vectors colored by the temperature. The 2 times zoom on the head (Obs. 14.1) shows the hot fluid being entrained inside the vortices, creating important mixing. The temperature in the core of the vortices is already close to $\left(T_{h}-T_{c}\right) / 2=62.5^{\circ} \mathrm{C}$ confirming this important mixing.

When the wave reaches the floor of the compartment, at $t=1 \mathrm{~s}$ (see Fig. 14 (b)), the head is broken, with the inner vortex (Obs. 14.2) moving toward the opening wall. At this time, the outer vortex has already detached itself from the main inflow and created a coherent structure moving towards the back wall (Obs. 14.3). This highly mixed vortex could be a real ignition threat, if it hits an ignition source such as an electric spark or a hot surface.

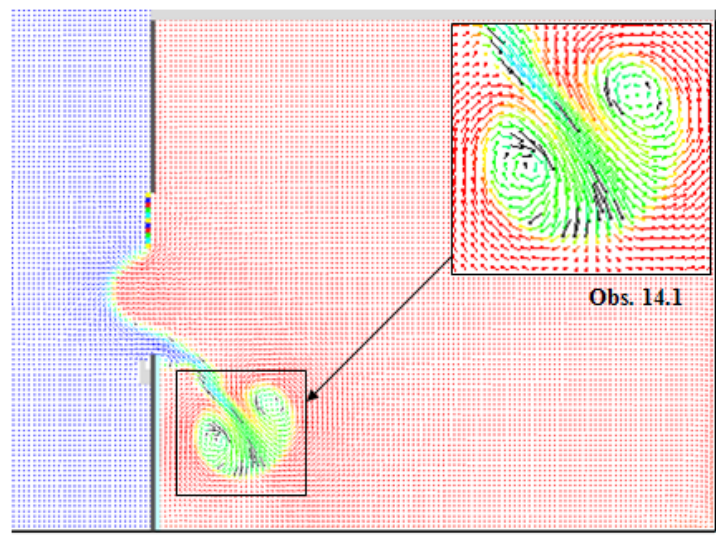

(a)

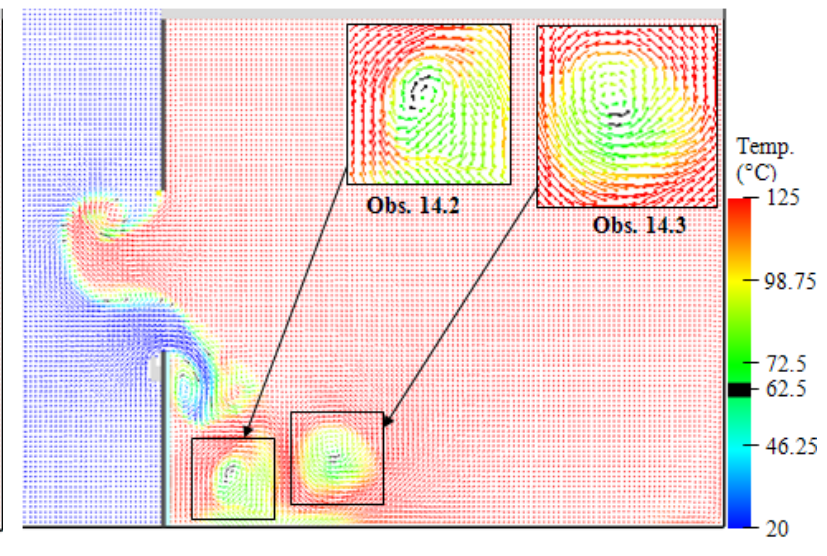

(b)

Fig. 14. Velocity vectors colored by temperature in middle opening configuration and $T_{h}=125^{\circ} \mathrm{C}$, at (a) $t=0.7 \mathrm{~s}$; (b) $t=1 \mathrm{~s}$ after opening, with 2 times zoom on the head of the wave.

After $t=1.3 \mathrm{~s}$ (see Fig. 15 (a)), the inner vortex moves up along the front wall creating a recirculation zone where cold air is entrained from the main flow, cooling this zone (Obs. 15.1). The outer vortex continues its way toward the back of the compartment, increasing its core temperature by entraining hot fluid.

After $t=1.7 \mathrm{~s}$ (see Fig. 15 (b)), the inner vortex hits the incoming flow and splits the wave (with a detached pocket of cold air, Obs. 15.2), enhancing the mixing and the cooling of the recirculation zone between the waterfall and the front wall. Meanwhile, the head of the wave has gained in temperature and has almost reached the back wall. 


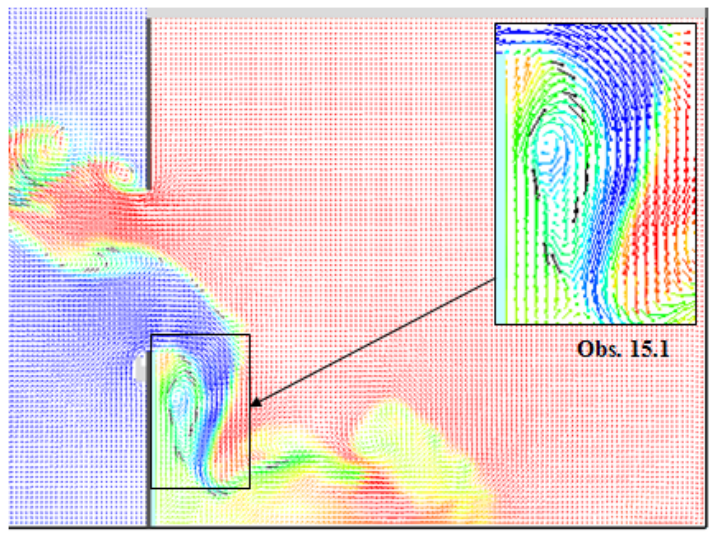

(a)

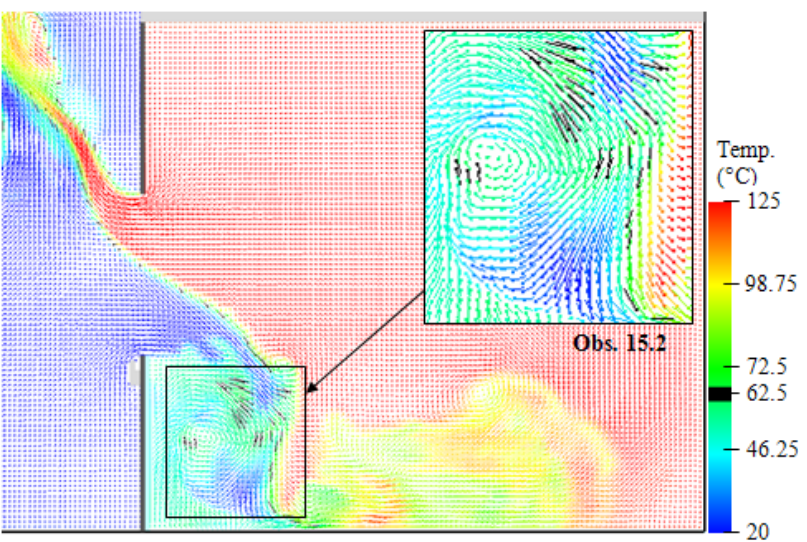

(b)

Fig. 15. Velocity vectors colored by temperature in middle opening configuration and $T_{h}=125^{\circ} \mathrm{C}$, at (a) $t=1.3 \mathrm{~s}$; (b) $t=1.7 \mathrm{~s}$ after opening, with 2 times zoom on the re-circulated inner vortex breaking the main flow.

\section{Bottom Opening Configuration}

In this section, we will discuss the flow after the opening. Figure 16 (a) shows the temperature contours at $t=1.6 \mathrm{~s}$. The head of the gravity wave is formed by a wide counter-rotating vortex, similar to the shape of the head of a forced flow (Obs. 16.1). The isotherm $T=40{ }^{\circ} \mathrm{C}$ is shown in black. It indicates that the head remains rather cool until the wave hits the back wall.

Figure 16 (b) shows the wave when it reaches the back wall. Some mixing is created by the rebound. Here we notice some Kelvin-Helmholtz instabilities (Obs. 16.2) observed previously by tomography, which are also responsible for some mixing. However, the mixing seems generally less important than in the middle opening configuration.

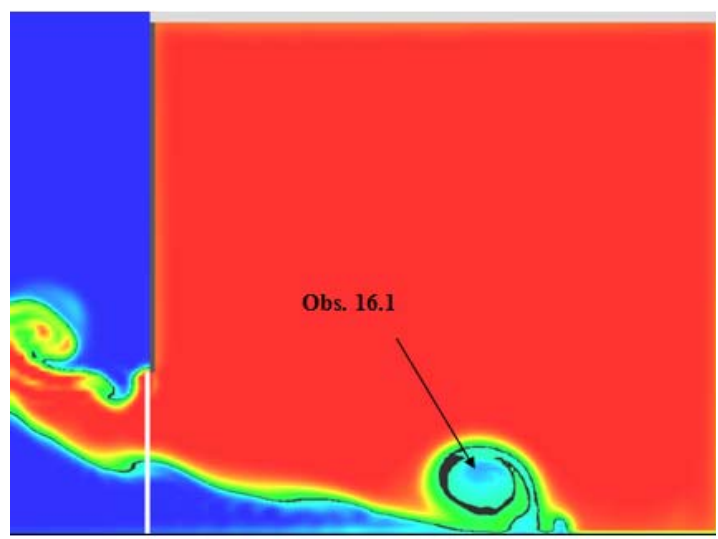

(a)

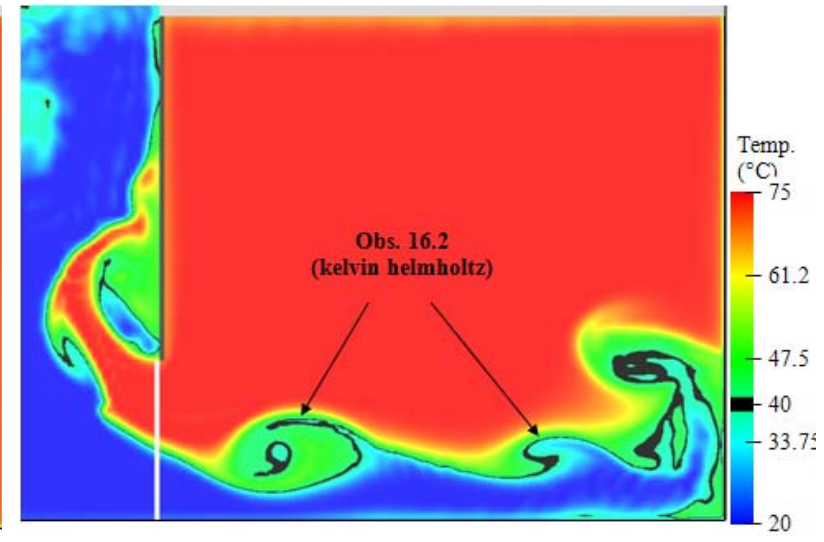

(b)

Fig. 16. Temperature contours in bottom opening configuration and $T_{h}=75^{\circ} \mathrm{C}$, at (a) $t=1.6 \mathrm{~s}$; (b) $t=3 \mathrm{~s}$ after opening.

\section{Quantification of the Mixing}

In order to estimate the mixing efficiency, we study the number of cells $N(\%)$ inside the compartment in which the mixing fraction $\mathrm{f}$ is between 0.4 and 0.6 . This condition will be referred to as $N[0.4<f<0.6]$.

Figure 17 shows the evolution of this number of cells as a function of time, for different initial hot temperatures. The time when the wave hits the back wall (rebound) and when it reaches the opening (back wave) is shown by the vertical lines, for $T_{h}=100{ }^{\circ} \mathrm{C}$. The intensification of mixing (maximum slope of $N(t)$ ) is at a maximum between the rebound and the back wave, and the peak value of mixing is just after the back wave, when the wave has just bumped into the inflow, and enhanced the mixing. Figure 18 shows 
the time average on $10 \mathrm{~s}$ and the maximum number of cells $N[0.4<f<0.6]$. It also shows the time the maximum is reached, the time to rebound and the time to back wave. It shows that the mixing level is maximum when the wave comes back. The maximum and average value of $N$ show that the mixing level is decreasing (except for $T_{\text {init }}=75^{\circ} \mathrm{C}$ ) when the density difference increases. It seems that the dominating cause for mixing enhancing is the effect of the 2 waves, inflow and back wave, crossing and bumping into each other close to the opening rather than the rebound on the back wall. Shortly after the time when the back wave occurs, the mixing level drops and then oscillates around $N=5 \%$.

It is interesting to note that the mixing process is much more intense and slower for $T_{h}=50{ }^{\circ} \mathrm{C}$. In the case of higher temperatures, the mixing level reaches its maximum value between rebound and back wave, showing that the rebound has more effect on mixing than for higher temperature differences. The slow motion of the gravity wave gives more time for the hot and cold layer to mix together and consequently the wave becomes thicker before hitting the back, which explains the more effective role played by the rebound on the mixing for this case.

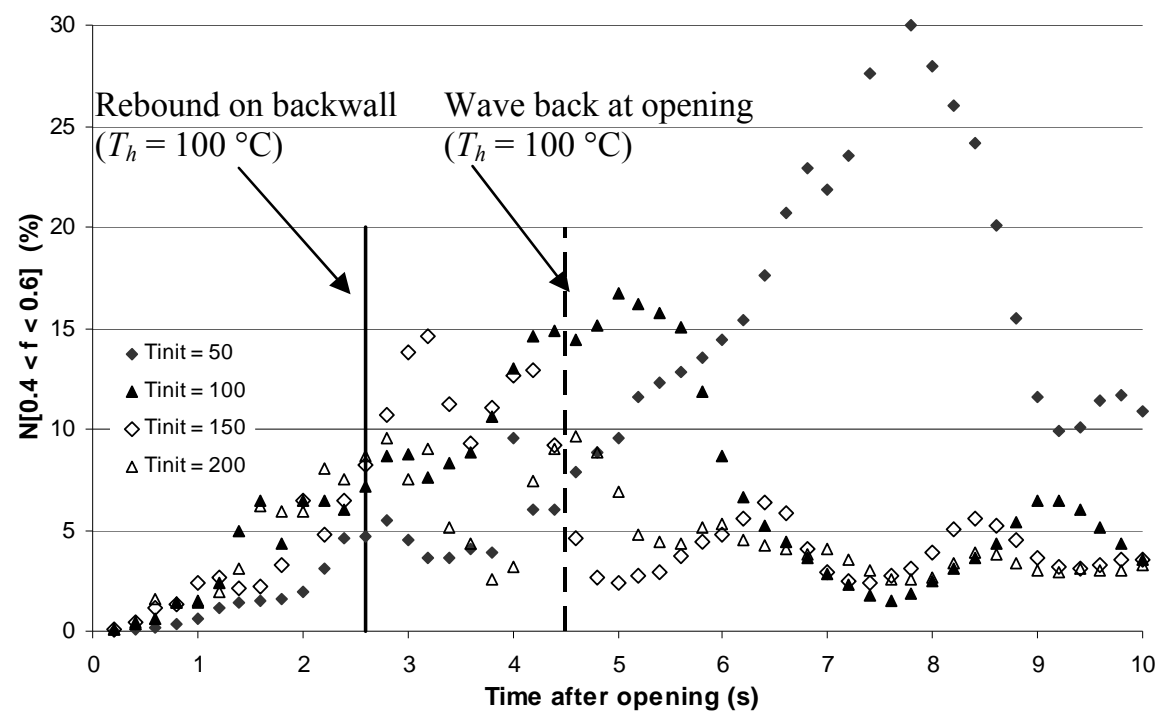

Fig. 17. Time evolution of the number of cells $N[0.4<f<0.6]$, for different initial temperature (middle opening configuration).

The same study is done for the bottom configuration, detailed in Ref. [1]. The dominant source of mixing depends on the opening configuration. When the opening is at the bottom, the main source is the rebound of the wave on the back wall. In the middle opening configuration, it is the bumping between the wave coming back from the wall after rebound and the inflow that causes a great deal to the mixing. 


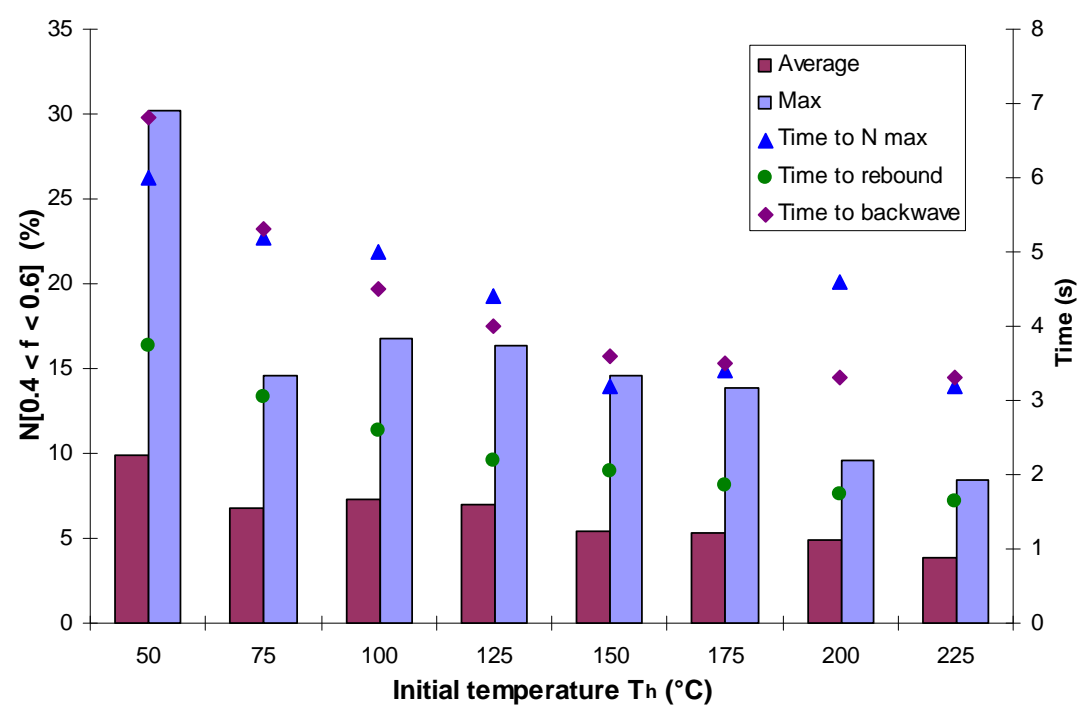

Fig. 18. Average and maximum values of $N[0.4<f<0.6]$ with the time to reach this maximum, the time to rebound and back wave for different initial temperature (middle opening configuration).

\section{CONCLUSION}

The experiments presented in this paper are the first to characterize the gravity wave in a non-isothermal gas phase in details. These preliminary results have shown a very unsteady flow, with high turbulence intensity. The shear stress zone between the two layers introduces the formation of vortices and of a pulsating character to the input flow. Previous preliminary numerical calculations didn't simulate this behavior, showing that current physical models of the CFD software cannot predict such a complex flow.

The experimental results were then used to calibrate and validate a numerical model. The qualitative comparison demonstrates that both methods show similar physical features of the mixing process. Quantitative results show good agreement between both methods. In addition, results were then used to validate theories of gravity wave developed by the authors. They seem to confirm a three phase behavior: an accelerating phase, a steady phase and a decay phase.

Once validated, the FDS model was used to investigate the mixing process between the two layers in greater detail. Ways to quantify this mixing were also considered. The results showed that coherent turbulent structures, such as counter-rotating vortices detaching from the waterfall in the middle opening configuration and Kelvin-Helmholtz instabilities in the bottom opening configuration, played an essential role in the mixing process. However, the mixing appeared generally less important in the bottom opening configuration than in the middle opening configuration. In the latter the first highly mixed detaching vortex could be a real ignition threat, if it were to hit an ignition source. The mixing quantification showed a higher mixing level in the middle opening configuration and that the dominant source of mixing was different depending on the configuration. It also showed that the mixing level is lower with higher density differences. It can be concluded that mixing is damped by acceleration.

Studying the hydrodynamic process of the mixing between fresh oxygen carried by a gravity wave with hot unburnt gases from an under-ventilated fire compartment is crucial in fire safety. These experimental and numerical results bring an understanding to the mixing process and will help to develop more efficient tactics to mitigate dangerous phenomena related to enclosure fires.

\section{REFERENCES}

[1] Guigay, G., A CFD and Experimental Investigation of Underventilated Compartment Fires, $\mathrm{PhD}$ Thesis, Department of Civil and Environmental Engineering, University of Iceland, 2008, http://www.skemman.is//handle/1946/3439

[2] Eliasson, J., Guigay, G.,and Karlsson, B., (2008) Enclosure fires, gravity waves, and the backdraft problem, Journal of Fire Sciences 26 (5): 373-397, http://dx.doi.org/10.1177/0734904108092116 
[3] Guigay, G., Eliasson, J., Karlsson, B., Horvat, A., and Sinai, Y., (2010) A Different Approach to Vent Flow Calculations in Fire Compartments using the Critical Flow Condition, Journal of Fire Sciences 28 (5): 409-439, http://dx.doi.org/10.1177/0734904109354966

[4] Fleischmann, M., and McGrattan, K.B., (1999) Numerical and Experimental Gravity Currents Related to Backdrafts, Fire Safety Journal 33 (1): 21-34, http://dx.doi.org/10.1016/S0379$\underline{7112(98) 00046-0}$

[5] Perez-Jimenez, C., Guigay, G., Karlsson, B., Eliasson, J., Horvat, A., Sinai, Y. and Franssen, J.M., (2009) Influence of Obstacles on the Development of Gravity Current Prior to Backdraft, Fire Technology 45 (3): 323-340, http://dx.doi.org/10.1007/s10694-008-0066-4

[6] Fleishmann, C.M., Backdraft phenomena, PhD Thesis, University of Berkley, NIST GCR 94 646, 1994.

[7] Fleishmann, C.M., Pagni, P., and Williamson, R, (1993) Exploratory backdraft experiments, Fire Technology 29 (4): 298-316, http://dx.doi.org/10.1007/BF01052526

[8] Guigay, G., Eliasson, J., Gojkovic, D., Bengtsson, L.G., and Karlsson, B., (2009) The Use of CFD Calculations to Evaluate Fire-Fighting Tactics in a Possible Backdraft Situation, Fire Technology 45 (3): 287-311, http://dx.doi.org/10.1007/s10694-008-0058-4

[9] Gottuk, D., (1999) The development and mitigation of backdraft: a real-scale shipboard study, Fire Technology 33 (4): 261-282, http://dx.doi.org/10.1016/S0379-7112(99)00033-8

[10] Chitty, R., “A Survey on Backdraught”, Pub No 5/94, Fire Research Station, Borehamwood, UK, 1994.

[11] Gorbett, G.E., and Hopkins, R., 'The current knowledge and training regarding backdraft, flashover and other rapid fire progression phenomena", NFPA world safety conference, Boston, 2007.

[12] Pedersen, F.B., Environmental Hydraulics: Stratified Flows, Springer-Verlag, Editions, Berlin Heidelberg, 1986.

[13] Anderson, J.D., Computational Fluid Dynamics, McGraw-Hill, New-York, 1995.

[14] Davidson, P.A., Turbulence, Oxford University Press, New York, 2004.

[15] Cushman-Roisin, B., (2005) Kelvin-Helmholtz Instability as a Boundary-value Problem, Environmental Fluid Mechanics 5 (6): 507-525, http://dx.doi.org/10.1007/s10652-005-2234-0

[16] McGrattan, K., Hostikka, S., Floyd, J., Baum, H., Rehm, R., Mell, W., and McDermott, R. "Fire Dynamics Simulator (Version 5) Technical Reference Guide. Volume 1: Mathematical Model," NIST Special Publication 1018-5, Gaithersburg, MD, 2010.

[17] McGrattan, K., Hostikka, S., Floyd, J., McDermott, R. "Fire Dynamics Simulator (Version 5) Technical Reference Guide Volume 3: Validation," NIST Special Publication 1018-5, Gaithersburg, MD, 2010. 